Communications in Physics, Vol. 26, No. 3 (2016), pp. 253-259

DOI:10.15625/0868-3166/26/3/8957

\title{
TRANSIENT ABSORPTION PHENOMENA IN SYNTHETIC HPHT AND CVD DIAMONDS FOR A FAST TIMING IN NUCLEAR INSTRUMENTATION
}

\author{
O. BUGANOV ${ }^{\dagger}$, S. TIKHOMIROV AND E. BARBARCHIK \\ B. I. Stepanov Institute of Physics, 220072, Minsk, pr. 60 Nezavisimosti \\ M. KORJIK, A. FEDOROV AND V. MECHINSKY \\ Institute for Nuclear Problems of BSU, 220030, Minsk, 11 Bobruiskaya \\ G. TAMULAITIS \\ Vilnius University, LT-10222, Vilnius, Saultekio 9-III \\ ${ }^{\dagger} E$-mail: o.buganov@ifanbel.bas-net.by
}

Received 05 December 2016

Accepted for publication 05 January 2017

\begin{abstract}
We investigate the transient absorption phenomena in synthetic diamonds obtained by High Pressure High Temperature (HPHT) and Chemical Vapor Deposition (CVD) methods. The aim of our study is to search inorganic crystalline media combining ionizing radiation and investigate their detecting properties and non-linear absorption of ultra-short laser pulses. The pump-probe technique with 140 fs laser pulses was used for the investigations.
\end{abstract}

Keywords: two-photon absorption, synthetic diamonds, absorption. from excited states, fast timing.

Classification numbers: 05.45.Tp, 78.47.J-, 81.05.ug.

\section{INTRODUCTION}

Search of the processes which will form time marks with precision better than $10 \mathrm{ps}$ and use the interaction of ionizing radiation with detecting media is a crucial demand for the development of a new generation of the detecting technique, especially in experimental particle and high energy physics. Recently we suggest using the sub-picosecond phenomena occurring just after the interaction of scintillating material with ionizing radiation for temporal mark formation by probing the material with ultra-short laser pulses [1-4].

(C)2016 Vietnam Academy of Science and Technology 
One of such phenomena is the elastic polarization due to the local lattice distortion caused by the displacements of electrons and holes generated by the ionization. This local lattice distortion results in redistribution of the density of states (DOS) of electron in the conduction band in the vicinity of the hole. The key feature of the elastic polarization is its ultrashort response time, which makes it prospective as an optically detectable time mark. Nonlinear optical absorption of femtosecond light pulses at appropriate wavelengths is considered to be a tool to form time mark. The effect of elastic polarization should be observed in many crystalline inorganic compounds. According to our estimations, the strongest effect should be observed in compounds with the bottom of the conduction band formed by $n \mathbf{d}$ orbitals of the lattice cations. In accordance with the crystal field theory, these orbitals are most sensitive to distortions of the crystal field in the vicinity of absorbing and emitting centers. Thus, the crystals with lattice cations having strong contribution of $\mathbf{d}$ orbitals in the conduction band (tungstates, molibdates, rare-earth and yttrium garnets, perovskites, oxy-orthosilicates, etc.) might be good candidates for time markers.

We confirmed the influence of ionization at $122 \mathrm{keV}$ on the two-photon two-color absorption of femtosecond laser pulses in $\mathrm{PbWO}_{4}$ crystals [1,3]. The observed effect encouraged our further study in this direction to develop a novel detecting technique exploiting the interaction of ultrashort laser pulses with crystalline media excited by ionizing radiation.

In this study, we investigate transient absorption phenomena in synthetic diamonds obtained by High Pressure High Temperature (HPHT) and Chemical Vapor Deposition (CVD) methods. Our study is to search inorganic crystalline media combining ionizing radiation and investigate their detecting properties and non-linear absorption of ultra-short laser pulses. Accordingly, we selected several diamond crystals suitable to detect ionizing radiation, particularly neutrons. Synthetic diamonds are now considered as the most prospective materials to operate in a harsh irradiation environment.

\section{AN ADVANTAGE OF THE TWO-PHOTON ABSORPTION TO PROBE FAST PROCESSES IN DETECTING MATERIALS}

One-photon absorption is extensively used to monitor different effects in ionizing radiation detectors. In fact, this technique enables monitoring slow changes in the detector material properties, particularly accumulation of the color centers under ionizing radiation. However, one-photon absorption is not convenient to explore changes in the transient ionizing radiation induced effects due to strong absorption of single photons via electronic transitions between valence and conduction bands. This is due to the origin of the bands of the majority of inorganic wide band gap compounds: p electronic states form the top of valence band, whereas $d$ and $f$ states of metal ions dominate in forming the bottom of the conduction band. Dipole-allowed $p-d$ transitions result in the absorption coefficient for the inter-band transitions at the order of $10^{5} \mathrm{~cm}^{-1}$.

The two-photon absorption coefficient is much less and intensity dependent. Consequently it can be adjusted by choosing of laser beam intensity and the photons absorbed via two-photon absorption propagate relatively long distances in the crystal. Two-photon absorption can involve photons of the same frequency generated by the same laser or simultaneously available photons of different frequencies [5]. The two-photon absorption involving one pump and one probe photon is a convenient tool for studying both temporal and spectral parameters of the inter band absorption. Recently, the pump-probe technique was exploited to study $\mathrm{PbWO}_{4}$ crystal $[1,3]$ which is the most popular detecting material in High Energy Physics Experiments at LHC CERN Program. 
The pump-induced changes in material properties resulting in modified probe absorption in the sample volume where the pump and probe beams spatially overlap have been recorded. Our measurements for 1-cm long samples showed that the change in optical density for the probe beam induced by the pump at $395 \mathrm{~nm}$ has a short rise time, its decay with characteristic time constant of few picoseconds, and a faster decay afterwards. The fast decrease of the induced absorption at the middle of the response curve was explained by the separation of the pump and probe beams in space, since the beams are focused on the same spot of the sample surface at slightly different angles. The duration of the response curve in this region depends also on the pump and probe wavelengths due to the dispersion of group light velocity.

\section{EXPERIMENTAL RESULTS}

Pump - probe setup was exploited in our study as described elsewhere $[3,6]$. The $140 \mathrm{fs}$ laser pulses of the second harmonic of $\mathrm{Al}_{2} \mathrm{O}_{3}: \mathrm{Ti}^{3+}$ laser was used to generate both the pump beam at a fixed central wavelength of $395 \mathrm{~nm}(3.15 \mathrm{eV})$ and a white continuum ranging from $400 \mathrm{~nm}$ to $1100 \mathrm{~nm}$, which was generated in water by a fraction of the fundamental laser beam at $790 \mathrm{~nm}$ and used as a probe. The diameters of the probe and pump spots within the sample were about 0.5 $\mathrm{mm}$ and $2 \mathrm{~mm}$, respectively.

The spectra of the signal probe beam and the reference one were recorded for every laser pulses by a polychromator equipped with silicon CCD matrix and transmitted to the computer. The differential absorbance signal $\Delta D(\lambda, \Delta t)$ is obtained as $\Delta D(\lambda, \Delta t)=\lg \left(\tilde{T}_{0} / \tilde{T}\right)$, where $\tilde{T}=$

$E_{\text {prob }} / E_{\text {ref }}$ and $\tilde{T}_{0}=E_{\text {prob }}^{0} / E_{\text {ref }}^{0}$ are energy ratios of the probe and reference pulses passed through the investigated sample at excitation and without it respectively. Typically, 30 measurements were averaged to give a wavelength-dependent signal at every time step. The transient absorption spectra are recorded in the range of $400 \mathrm{~nm}-800 \mathrm{~nm}$ with a resolution of $1.5 \mathrm{~nm}$. The estimated absolute error for $\Delta D$ was as about 0.001 . The transient spectra were corrected for the group velocity dispersion of the probe pulse

Two High Pressure High Temperature (HPHT) diamond samples were produced by ADAMAS-BSU, Minsk, Belarus. The sample dimensions are $4 \times 4 \times 0.3 \mathrm{~mm}$. The samples had yellow coloration due to the presence of nitrogen. The cutoff of the transmission spectrum of both samples was found to be near $420 \mathrm{~nm}$. One of the samples was found to be scintillating, and another to be not scintillating. The Chemical Vapor Deposition (CVD) diamond with dimensions of $4 \times 2 \times 0.15 \mathrm{~mm}$ was provided by CERN.

In contrary to the $\mathrm{PbWO}_{4}$ complex oxide crystal based on oxy-anionic complexes, diamond is a wide band gap indirect semiconductor having a relatively simple electronic band structure due to its monoatomic network. Two-photon absorption in nitrogen-free diamond was investigated in detail [7]. The lowest two-photon absorption band was observed to a peak at the total two-photon energy of $5.5 \mathrm{eV}$. This energy corresponds to the indirect transition to conduction band. The direct two-photon absorption was found to occur at $6.5 \mathrm{eV}$.

We observed two-photon absorption effects in all diamond samples under the study. Figures 1, 2 and 3 show our obtained results. The most important feature for the response of all studied samples is that the leading edge of the differential absorption is limited by laser pulse shape rather than by material properties. Thus, the processes responsible for the nonlinear absorption occur on femtosecond time domain. 
a)
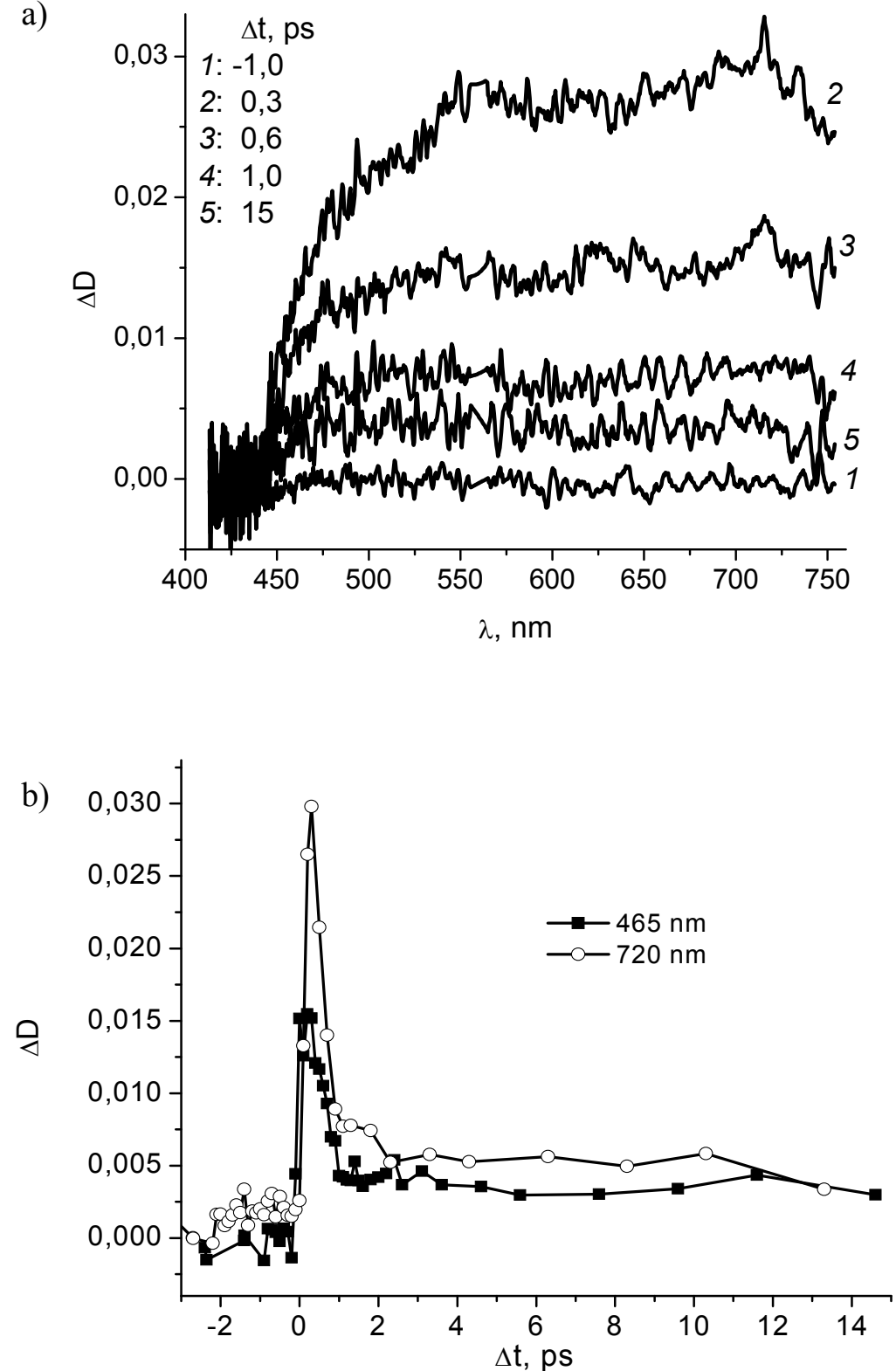

Fig. 1. a) Spectrum of differential absorption of the no-scintillating diamond sample at $395 \mathrm{~nm}$ pump at different delays between pump and probe (indicated); b) Kinetics of differential absorption of the no-scintillating diamond sample at $395 \mathrm{~nm}$ pump and two probe wavelengths: $465 \mathrm{~nm}$ and $720 \mathrm{~nm}$. 

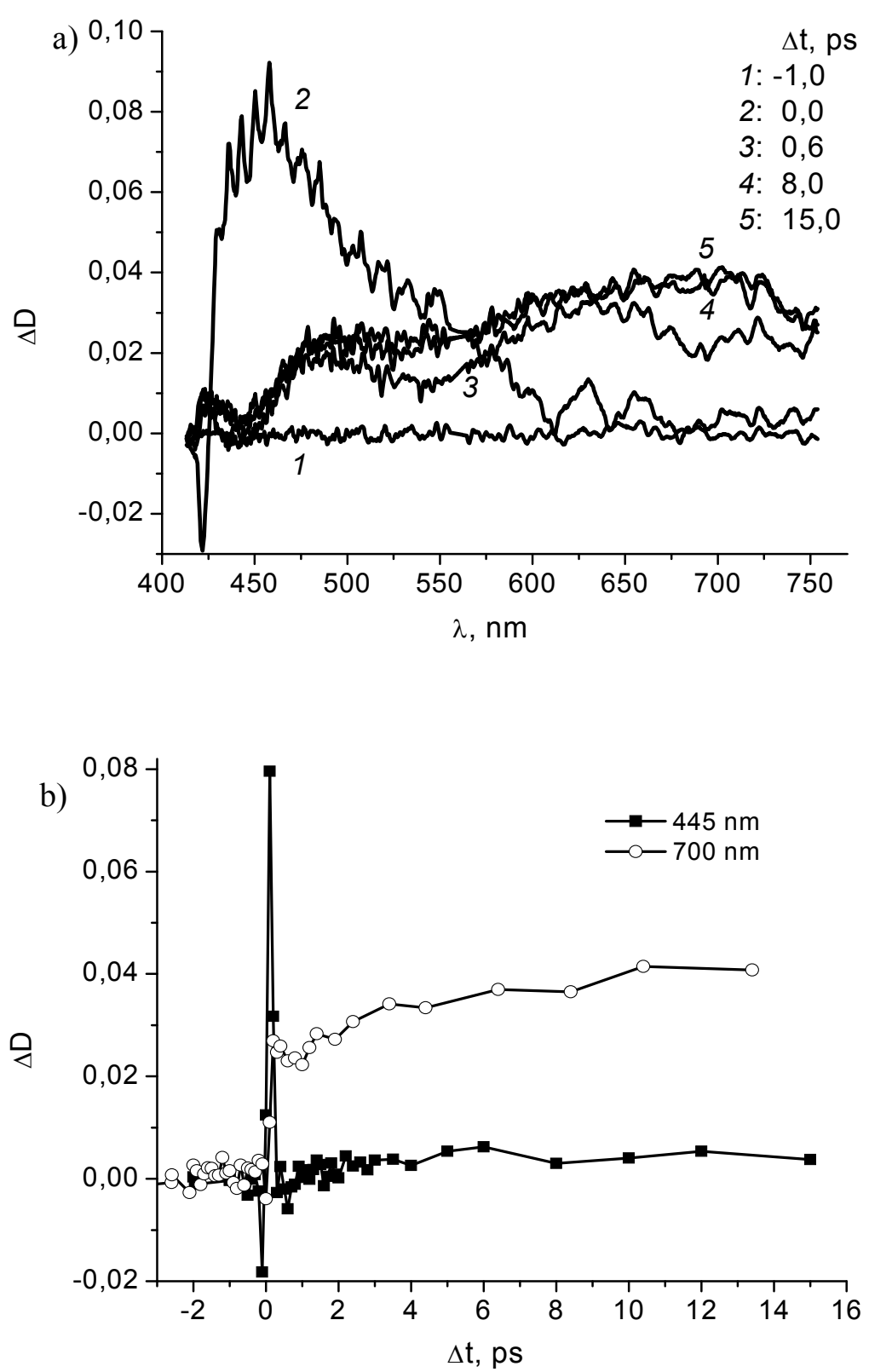

Fig. 2. a) Spectrum of differential absorption of the scintillating diamond sample at $395 \mathrm{~nm}$ pump at different delays between pump and probe (indicated); b) Kinetics of differential absorption of the scintillating diamond sample at $395 \mathrm{~nm}$ pump and two probe wavelengths: $465 \mathrm{~nm}$ and $720 \mathrm{~nm}$. 

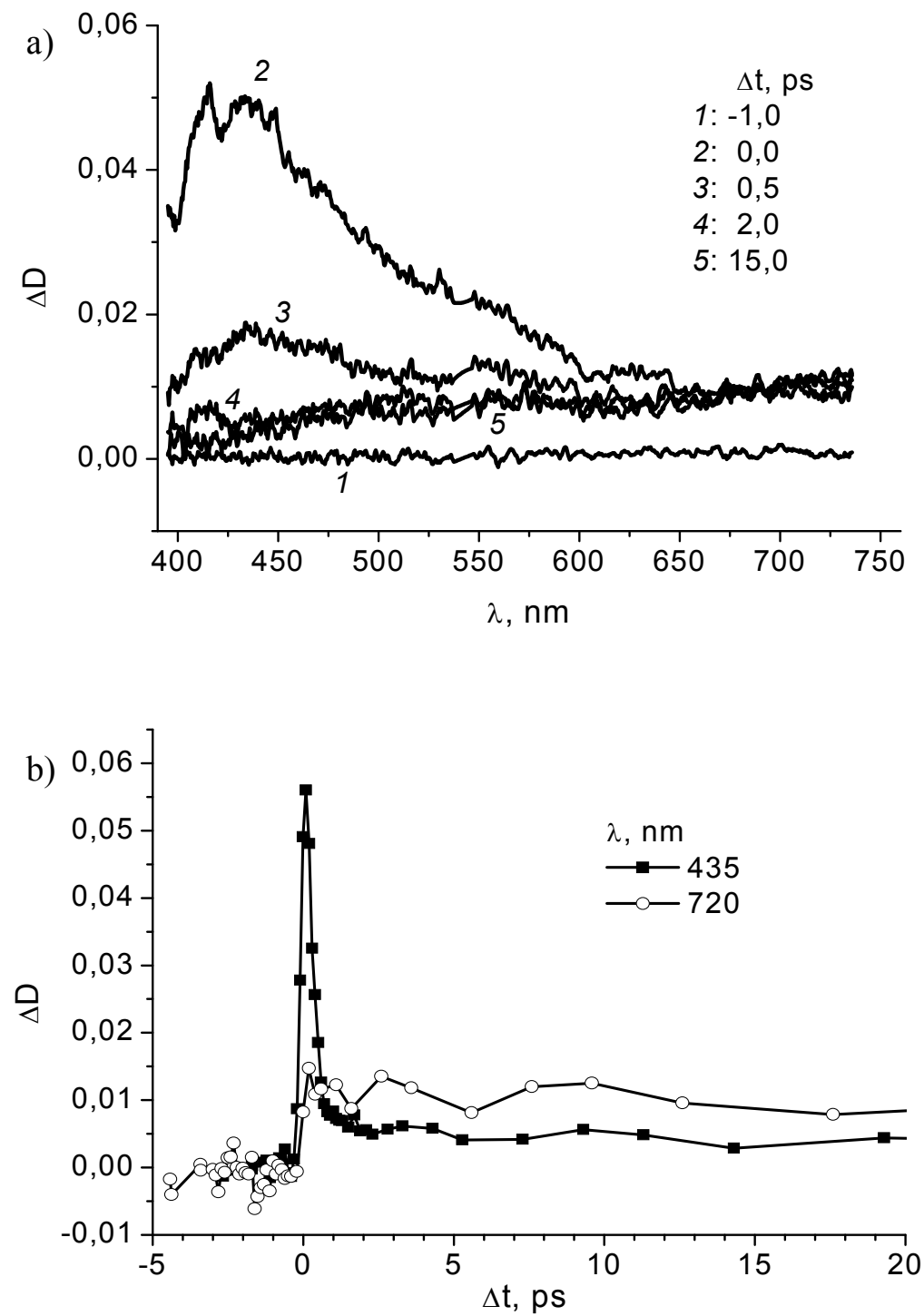

Fig. 3. a) Spectrum of differential absorption of the CVD diamond sample at $264 \mathrm{~nm}$ pump at different delays between pump and probe (indicated); b) Kinetics of differential absorption of scintillating diamond sample at $263 \mathrm{~nm}$ pump and two probe wavelengths: $435 \mathrm{~nm}$ and $720 \mathrm{~nm}$.

The band peaked at $\sim 465 \mathrm{~nm}(2.6 \mathrm{eV})$ correlates fairly well with the energy of the indirect two-photon two-color transition (one photon from pump pulse and other - from probe) to the conduction band. Other bands observed in the samples are peaked within $550-600 \mathrm{~nm}$ and at $\sim 700 \mathrm{~nm}$. All induced absorption bands decay fast within the first picoseconds and have also a slow decay component. The total energy of pump and probe photons for the $550-600 \mathrm{~nm}$ and 
$700 \mathrm{~nm}$ bands are considerably smaller than band gap of diamond, so we can conclude that defect centers are mainly responsible for the transient absorption in this spectral region.

It should be noted that when the scintillating diamond sample is probed at $700 \mathrm{~nm}$, the kinetics of transient absorption (Fig. 2) is differ from that of the no-scintillating sample (Fig. 1). There are two risen processes: the fast process, less than one picosecond, is dedicated to direct excitation of defects by two-photon or one- photon transitions and the slow process lasts a few ps, which to our mind, is due to a population of the defects by capturing the carriers from conduction zone.

On the contrary to the HPHT diamond samples, the CVD diamond did not show any spectra of differential absorption at $395 \mathrm{~nm}$ pump. Strong differential absorption was found only at pump $264 \mathrm{~nm}$. We observed a strong band in the blue range peaked at $430 \mathrm{~nm}$ and also two weak bands roughly peaked at $\sim 550 \mathrm{~nm}$ and $700 \mathrm{~nm}$ (Fig. 3). So, one can state that two last bands related with the defects and that concentration of defect centers in the HPHT diamonds is considerably larger than that in the CVD diamond.

The difference in transient absorption decay is observed for the CVD diamond with the probe at $435 \mathrm{~nm}$ and $720 \mathrm{~nm}$ as seen in Figure 3 (b). Due to small concentration of defects the signal is relatively weak with the probe at $720 \mathrm{~nm}$ but has an increasing tendency toward the IR region.

\section{CONCLUSION}

The observed nonlinear optical effects might be exploited for timing of the interaction of diamond detector materials with ionizing radiation by one-photon absorption or by two-photon

absorption methods. Two-photon two-color absorption has the advantages in sensitivity, time resolution, and simplicity of signal interpretation.

\section{ACKNOWLEDGEMENT}

The partial financial support from Belarusian Republican Foundation for Fundamental Research (Project F16-011/1, Project F16V2-003) and MSC INTELUM project are appreciated.

\section{REFERENCES}

[1] E. Auffray, O. Buganov, M. Korjik, A. Fedorov, S. Nargelas, G. Tamulaitis, S. Tikhomirov, A. Vaitkevicius, Nucl. Instr. Meth. Phys. Res. A 804 (2015) 194.

[2] E. Auffray, O. Buganov, A. Fedorov, M. Korjik, P. Lecoq, G. Tamulaitis, S. Tikhomirov, A. Vasil'ev, Journal of Physics: Conf. Series 587 (2015) 012056.

[3] E. Auffray, O.V. Buganov, M. V. Korjik, S. A. Tikhomirov, A. A. Fedorov, A. D. Shirokanov, Vesti NAS of Belarus, Ser. Phys.-Math (Rus) No. 3 (2015) 91 (in Belarusian).

[4] M. Korjik, E. Auffray, O. Buganov, A. Fedorov, I. Emelianchik, E. Griesmayer, V. Mechinsky, S. Nargelas, O. Sidletskiy, G. Tamulaitis, S. Tikhomirov, A. Vaitkevicius, EEE Transactions on Nuclear Science 63 (2016) 2979-2984.

[5] J.-C. Diels and W. Rudolph, Ultrashort Laser Pulse Phenomena, Elsevier, 2006, p. 647.

[6] Vitali I. Stsiapura, Siarhei A. Kurhuzenkau, Valery A. Kuzmitsky, Oleg V. Bouganov and Sergey A. Tikhomirov, J. Phys. Chem. A 120 (2016) 5481-5496.

[7] T. Roth, R. Raenen, Opt. Commun. 189 (2001) 289-296. 